\title{
Gallstone ileus with fistula: an unusual case report
}

\author{
Leon Agung Manurung ${ }^{1}$, Prijambodo ${ }^{2}$ \\ leonagungmanurung@gmail.com \\ ${ }^{1}$ Radiologist, Department of Radiology, Faculty of Medicine, Airlangga University, Surabaya, \\ Indonesia \\ ${ }^{2}$ Radiologist Consultant, Department of Radiology, Faculty of Medicine, Airlangga University, \\ Surabaya, Indonesia
}

\begin{abstract}
:
Background: Gallstone ileus is a very uncommon consequence of cholelithiasis, which is defined as mechanical intestinal obstruction caused by one or more large gallstones impaction in the digestive tract. It is caused by gallstones passing through the fistula from the bile duct into the intestinal lumen. The most frequently encountered type of fistula is between the gallbladder and the duodenum

Case Presentation: A 63-year-old male presented to our emergency department with severe abdominal pain, nausea, and vomiting. For seven days prior to the onset of the illness, the patient experienced periumbilical abdominal pain with no apparent cause. Contrast-enhanced abdominal CT scan revealed gallstones ileus with stones with a diameter of $+/-2.3 \mathrm{~cm}$ in the ileum and a suspicion of a fistula between the gallbladder and the duodenum, as well as between the gallbladder and the transverse colon. After that, the patient underwent emergency room surgery for a laparotomy. Then, the patient was admitted to the intensive observation room for one week and received intensive care. Throughout treatment, the patient's condition deteriorated due to sepsis-related complications and comorbidities.
\end{abstract}

Conclusion: Rigler's triad (biliary tract obstruction, intestinal obstruction, and ectopic stones) is the most widely accepted imaging diagnostic criterion.

Keyword: Gallstone ileus; Fistula; X-Ray; Abdominal CT scan

\section{Introduction.}

Gallstone ileus is a very uncommon consequence of cholelithiasis, which is defined as mechanical intestinal obstruction caused by one or more large gallstones impaction in the digestive tract. It is caused by gallstones passing through the fistula from the bile duct into the intestinal lumen. The most frequently encountered type of fistula is between the gallbladder and the duodenum. Once a stone has entered the intestinal lumen, it can obstruct any section of the digestive tract, but the distal ileum is the most typical location (Ploneda-Valencia et al., 2017).

\section{Case Report.}

On September 6, 2020, a 63-year-old male presented to our emergency department with severe abdominal pain, nausea, and vomiting. For seven days prior to the onset of the illness, the patient experienced periumbilical abdominal pain with no apparent cause. The pain was described as chronic dullness interspersed with episodes of more severe pain, and it was accompanied by nausea, vomiting, bowel obstruction, and defecation.

Abdominal distension marked tenderness in the middle and upper abdomen, mild muscle tension, and hyperactive bowel sounds were noted on physical examination. Given the patient's history of gallstone disease, gallstone ileus was suspected as the cause of the patient's symptoms. Following that, the patient was examined in the supine and lld (left lateral decubitus) position for abdominal radiographs. Small bowel obstruction was discovered via conventional X-ray examination, with the appearance of stones in the ileum (Figure 1). The surgical operator then requested a contrast-enhanced CT scan of the abdomen, which revealed gallstones ileus with stones with a diameter of $+/-2.3 \mathrm{~cm}$ in the ileum and a suspicion of a fistula between the gallbladder and the duodenum, as well as between the gallbladder and the transverse colon (Figure 2,3). 

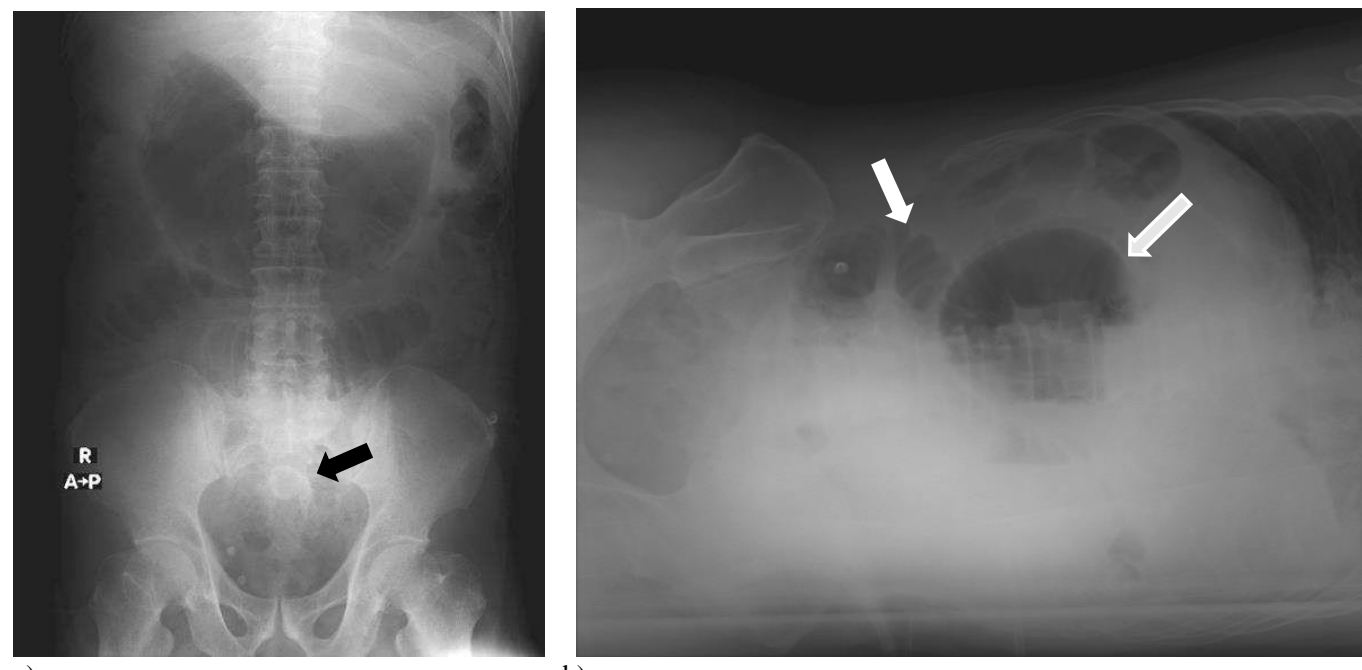

b)

Figure 1. Abdominal Plain Photo : Supine and lld. a) A radiopaque shadow measuring $+/-2.3 \mathrm{~cm}$ is visible in the central abdominal region, indicating the presence of gallstones; b) Dilatation of the small bowel shadow in the central abdominal region with a coiled spring appearance.
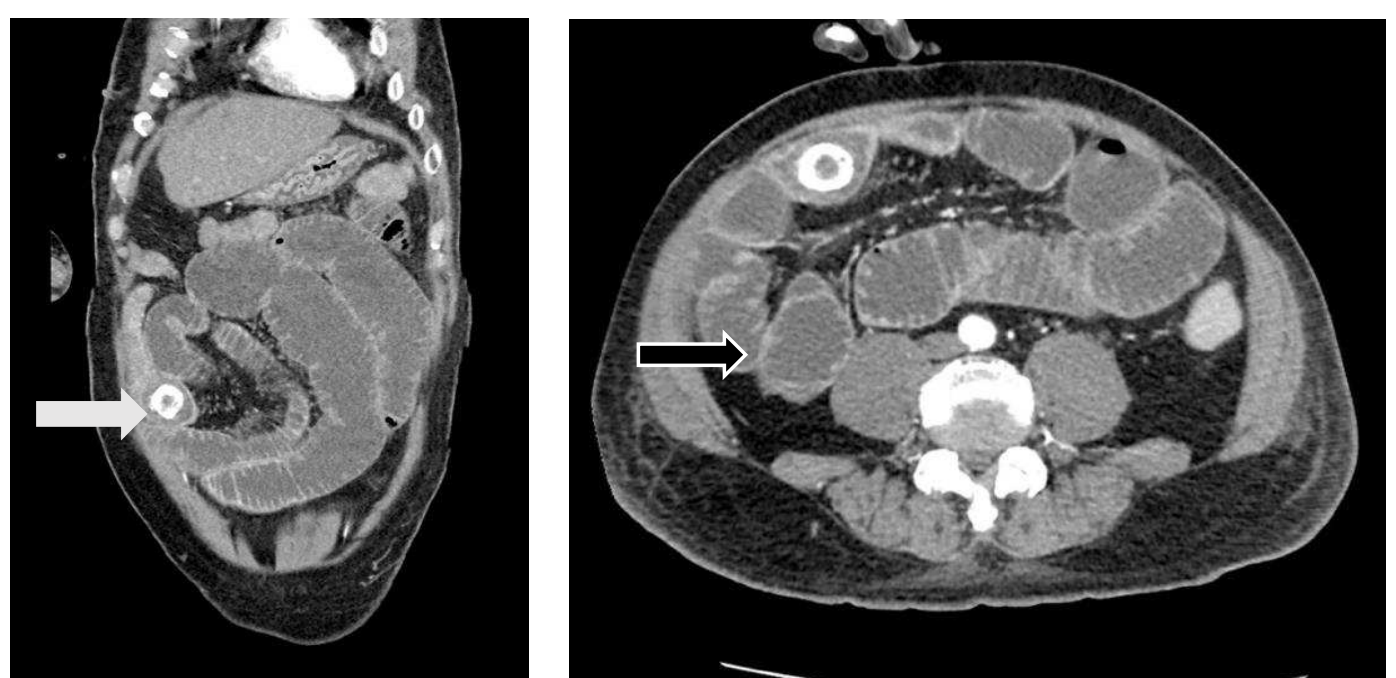

Figure 2. Enhanced abdominal CT scan axial and coronal slices: There are stones with a diameter of $+/-2.3 \mathrm{~cm}$ that originate in the bile and are associated with small bowel dilatation. 


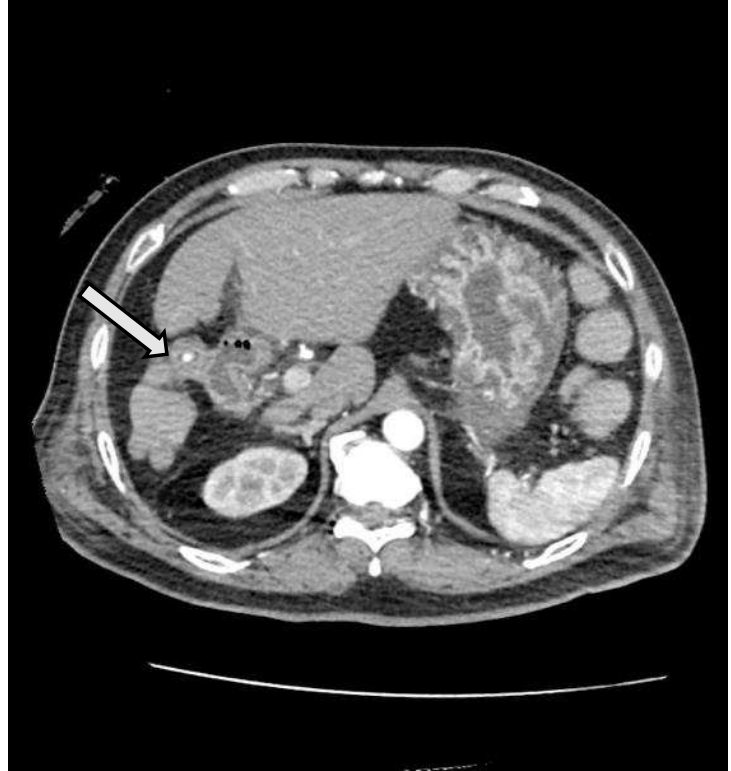

Figure 3. a) Enhanced abdominal CT scan axial slices: There is a fistulation between the transverse colon and the gall bladder with stones in it; b) Enhanced abdominal CT scan coronal slices: Between the duodenum part 1 and the gallbladder, a fistula develops.

After that, the patient underwent emergency room surgery for a laparotomy. The jejunum and ileum were found to be dilated up to $70 \mathrm{~cm}$ proximal to the ileocaecal junction, with stones measuring 3 $\mathrm{cm}$ in diameter and dark in colour. Additionally, the gallbladder, transverse colon, and duodenum were conglomerated, with a gallbladder-duodenum fistulation part 1 diameter of $2 \mathrm{~cm}$ and a gallbladder-colon transverse diameter of $2 \mathrm{~cm}$. Then, stone extraction, adhesiolysis conglomeration, fistula excision, and duodenal and colon repair were performed. The patient was admitted to the intensive observation room for one week and received intensive care. Throughout treatment, the patient's condition deteriorated due to sepsis-related complications and comorbidities.

\section{Discussion}

Cholecystoduodenal fistula associated with gallstone ileus is a clinical entity that occurs infrequently. It is frequently caused by uncontrolled gallstones and chronic cholecystitis for an extended period of time. Gallstone ileus can manifest itself in any part of the gastrointestinal tract, but the terminal ileum and ileocecal valve are the most frequently obstructed areas (Reisner \& Cohen, 1994).

Rigler's triad is the gold standard imaging diagnostic criteria: biliary tract obstruction, intestinal obstruction, and ectopic stones. Over $50 \%$ of patients exhibit two or more of the triads (Kirchmayr et al., 2005). This patient was hospitalized due to an intestinal obstruction. Cholestasis and ectopic stones were discovered during a CT examination, and the diagnosis was confirmed following a thorough review of the patient's previous gallstone history.

Surgery is preferred for treatment, and the surgical approach should be customized for each case (de Alencastro et al., 2013). The treatment is based on the principle of removing the gallstone, gallbladder, cutting the fistula, and repairing the fistula. The major surgical procedures are as follows: 1) simple surgical incision into the intestine and stone extraction; 2) multiple-stage incision into the intestines to remove the stone during emergency treatment, gallbladder removal, and fistula repair; 3) single-stage incision into the intestines to remove the stone, gallbladder removal, and fistula repair. The most frequently reported surgical procedure is a simple intestine incision and stone removal. The advantage of this procedure is that it is a straightforward procedure that requires little time and has a low overall mortality rate. The disadvantage is that it is ineffective in treating biliary tract disease, which increases the risk of biliary tract infection and gallbladder cancer following surgery (Shioi et al., 2012). The procedure, which includes an incision into the intestines to remove the stone, gallbladder removal, and fistula repair in a single stage, is comprehensive in terms of disease treatment, but patients must be able to tolerate the surgery. However, the disease 
frequently affects elderly patients (Vasilescu et al., 2013), who have a difficult time enduring a lengthy and complicated surgery.

\section{References}

de Alencastro, M. C., Cardoso, K. T., Mendes, C. A., Boteon, Y. L., de Carvalho, R. B., \& Fraga, G. P. (2013). Acute intestinal obstruction due to gallstone ileus. Revista Do Colégio Brasileiro de Cirurgiões, 40(4), 275-280. https://doi.org/10.1590/S0100-69912013000400004

Kirchmayr, W., Mühlmann, G., Zitt, M., Bodner, J., Weiss, H., \& Klaus, A. (2005). Gallstone ileus: Rare and still controversial. ANZ Journal of Surgery, 75(4), 234-238. https://doi.org/10.1111/j.1445-2197.2005.03368.x

Ploneda-Valencia, C. F., Gallo-Morales, M., Rinchon, C., Navarro-Muñiz, E., Bautista-López, C. A., de la Cerda-Trujillo, L. F., ReaAzpeitia, L. A., \& López-Lizarraga, C. R. (2017). Gallstone ileus: An overview of the literature. Revista de Gastroenterología de México (English Edition), 82(3), 248-254. https://doi.org/10.1016/j.rgmxen.2017.05.001

Reisner, R. M., \& Cohen, J. R. (1994). Gallstone ileus: a review of 1001 reported cases. The American Surgeon, 60(6), 441-446.

Shioi, Y., Kawamura, S., Kanno, K., Nishinari, Y., Ikeda, K., Noro, A., \& Kooka, F. (2012). A case of gallstone ileus displaying spontaneous closure of cholecystoduodenal fistula after enterolithotomy. International Journal of Surgery Case Reports, 3(1), 12-15. https://doi.org/10.1016/j.ijscr.2011.07.012

Vasilescu, A., Cotea, E., Palaghia, M., Vintilă, D., \& Târcoveanu, F. E. (2013). Gallstone ileus: a rare cause of intestinal obstruction -- case report and literature review. Chirurgia (Bucharest, Romania : 1990), 108(5), 741-744. 